\title{
Teamwork and Delegation in Medical Homes: Primary Care Staff Perspectives in the Veterans Health Administration
}

\author{
Gala True, PhD ${ }^{1,2}$, Greg L. Stewart, PhD ${ }^{3,4}$, Michelle Lampman, MA ${ }^{3,5}$, Mary Pelak, MSW', and \\ Samantha L. Solimeo, PhD, MPH 3.5 \\ 'VISN 4 Center for Evaluation of Patient Aligned Care Teams (CEPACD), Philadelphia Veterans Affairs Medical Center, Philadelphia, PA, USA; \\ ${ }^{2}$ University of Pennsylvania Perelman School of Medicine, Philadelphia, PA, USA; ${ }^{3}$ Department of Veterans Affairs, VISN23 Patient \\ Aligned Care Team Demonstration Lab, lowa City VA Health Care System, lowa City, IA, USA; ${ }^{4}$ Tippie College of Business, University of \\ lowa, lowa City, IA, USA; ${ }^{5}$ Department of Veterans Affairs, Center for Comprehensive Access \& Delivery Research and Evaluation \\ (CADRE), lowa City VA Health Care System, lowa City, IA, USA.
}

BACKGROUND: The patient-centered medical home $(\mathrm{PCMH})$ relies on a team approach to patient care. For organizations engaged in transitioning to a $\mathrm{PCMH}$ model, identifying and providing the resources needed to promote team functioning is essential.

OBJECTIVE: To describe team-level resources required to support $\mathrm{PCMH}$ team functioning within the Veterans Health Administration (VHA), and provide insight into how the presence or absence of these resources facilitates or impedes within-team delegation.

DESIGN: Semi-structured interviews with members of pilot teams engaged in $\mathrm{PCMH}$ implementation in 77 primary care clinics serving over 300,000 patients across two VHA regions covering the Mid-Atlantic and Midwest United States.

PARTICIPANTS: A purposive sample of 101 core members of pilot teams, including 32 primary care providers, 42 registered nurse care managers, 15 clinical associates, and 12 clerical associates.

APPROACH: Investigators from two evaluation sites interviewed frontline primary care staff separately, and then collaborated on joint analysis of parallel data to develop a broad, comprehensive understanding of global themes impacting team functioning and within-team delegation.

KEY RESULTS: We describe four themes key to understanding how resources at the team level supported ability of primary care staff to work as effective, engaged teams. Team-based task delegation was facilitated by demarcated boundaries and collective identity; shared goals and sense of purpose; mature and open communication characterized by psychological safety; and ongoing, intentional role negotiation.

CONCLUSIONS: Our findings provide a framework for organizations to identify assets already in place to support team functioning, as well as areas in need of improvement. For teams struggling to make practice changes, our results indicate key areas where they may benefit from future support. In addition, this research sheds light on how variation in medical home implementation and outcomes may be associated with variation in team-based task delegation.

Published online April 9, 2014
KEY WORDS: patient-centered medical home; teams; implementation; Veteran's health; qualitative evaluation; primary care; Department of Veterans Affairs.

J Gen Intern Med 29(Suppl 2):S632-9

DOI: $10.1007 / \mathrm{s} 11606-013-2666-\mathrm{Z}$

(C) Society of General Internal Medicine 2013

\section{BACKGROUND}

The patient-centered medical home (PCMH) has been proposed as a solution to multiple problems in U.S. healthcare: rising costs and disparities, ${ }^{1}$ growing needs of an aging population with chronic and complex illnesses, ${ }^{2}$ and a projected shortage of primary care physicians. ${ }^{3}$ Demonstration projects in a variety of practice settings have evaluated process and outcome-related effects of the PCMH, such as changes in processes of care, ${ }^{4-6}$ quality, ${ }^{7,8}$ cost,,${ }^{9,10}$ utilization, ${ }^{10-13}$ and patient experience. ${ }^{14-16}$ Recent evaluations of national PCMH initiatives have recommended close examination of primary care staff implementation experiences with an eye towards provider and staff role transformation. ${ }^{17,18}$

Role transformation is an integral component of PCMH team function and has emerged as a key topic of inquiry. ${ }^{19}$ The PCMH is built on a team approach, wherein responsibility for patient care is distributed among an interdisciplinary team performing health care tasks interdependently, rather than operating in professional silos. ${ }^{20,21}$ Team-based task delegation has been described as essential to primary care providers' (PCPs') capacity to provide recommended preventive and chronic care services to patients. ${ }^{22}$ However, little is understood about the conditions that foster or impede delegation in the context of the PCMH model. Delegation is not merely the reassignment of work tasks from one team member to another. Rather, delegation is a mind-set requiring PCPs to embrace the idea that sharing aspects of patient care with other team members will result in higher quality care and greater efficiency, as well as a climate in which team members adopting new responsibilities feel empowered and clear about their roles. ${ }^{23}$ For 
organizations engaged in transitioning to a PCMH model, identifying and providing the resources needed to support teamwork, particularly within-team delegation, is essential to gaining and sustaining forward momentum. ${ }^{24}$

The Veterans Health Administration (VHA) has undertaken the nation's largest-scale transformation to a PCMH or Patient Aligned Care Team (PACT) model, with over 7,000 primary care teams slated to operate as PACTs by $2014 .{ }^{25}$ With the funding of five Demonstration Laboratories (Labs) and a Coordinating Center, VHA has also invested in a comprehensive and longitudinal evaluation of PACT implementation across representative geographic and demographic regions. Each Lab is charged with assessing PACT within a defined region, which ensures sensitivity to context-specific variations, such as rurality, facility complexity, implementation climate, and veteran demographics. Cross-Lab collaboration provides opportunities to identify national trends: this paper reports findings from one such collaboration across two Labs.

Our prior work describes system-level challenges encountered by pilot teams, namely discordant or unsupportive administrative leadership, lack of access to timely and accurate data, and staffing issues. ${ }^{26,27}$ Striking similarities in our findings, despite significant geographic variation across sites, led to cross-Lab conversations about the conditions necessary to form and support effective, engaged teams in medical homes. Accordingly, we conducted a joint analysis of interview data to identify resources that help frontline primary care staff function as teams. By resources we mean support, assets, and infrastructure that can be drawn upon when needed. In contrast to our earlier studies focusing on system and clinic characteristics, in this study we sought to characterize internal, team-level resources, and to gain insight into how the presence or absence of these resources facilitate or impede within-team delegation.

\section{METHODS}

Although we study PACT implementation across two regions (called Veterans Integrated Service Networks, or VISNs) with considerable variation, we share common methodology and research goals. The VISN 4 Lab evaluates PACT implementation in 56 primary care clinics located across six states in the Mid-Atlantic. The VISN $23 \mathrm{Lab}$ has focused on the experiences of 22 primary care teams distributed across the VA Midwest Healthcare Network located in the central U.S. Both Labs are conducting a mixed-methods practical formative evaluation ${ }^{28}$ to understand factors affecting variation in implementation, as well as experiences and perspectives of frontline primary care staff. ${ }^{26,27}$ Within the practical formative evaluation framework, each Lab employs a variety of individual methods, including observational site visits, review of internal organizational documents, interview techniques, and feedback mechanisms. ${ }^{28}$ Details of the overall study design for both Labs are described elsewhere. ${ }^{26,27}$ Methodological similarities enable a collaborative, joint analysis of parallel qualitative data across the two Labs in order to demonstrate transferability of findings and to develop a broad, comprehensive understanding of the challenges facing PACTs nationally.

\section{Design}

To understand and characterize resources required for primary care staff to function as medical home teams, investigators from each Lab conducted semi-structured interviews during an 18month pilot phase (June 2010 through December 2011). Interviews with frontline PACT members in VISN 4 were conducted in-person during site visits or at training events, or over the telephone. Interviews in VISN 23 were conducted by telephone. Although developed separately by investigators with each Lab, interview guides used in both VISNs covered similar topics including: efforts to improve practice processes and outcomes; team roles and expectations for different team members; and challenges arising during PACT implementation. Interviews averaged $45 \mathrm{~min}$ in length and all interviews were audio-recorded and transcribed. VISN 23 Lab's evaluation activities were reviewed and approved as research by the Iowa City VA Healthcare System Institutional Review Board (IRB) and Research and Development Committee, while those of the VISN 4 Lab were reviewed and approved as Quality Improvement by the Philadelphia VA Medical Center IRB.

\section{Participants}

In the VHA model there are four core members of a PACT. These include a physician, physician assistant, or nurse practitioner (PCP); a registered nurse who serves as a care manager (RNCM); a licensed practical nurse (Clinical Associate); and a Clerical Associate who typically does not have formal medical training. We interviewed a purposive sample of 101 members of PACT pilot teams across VISNs 4 and 23, including PCPs $(n=32)$, RNCMs $(n=42)$, Clinical Associates $(n=15)$, and Clerical Associates $(n=12)$. Respondents were identified through internal documents listing members of pilot teams or via correspondence with key contacts at VHA primary care clinics. Recruitment for interview participation was conducted by e-mail correspondence, during site visits, or at PACT training events.

\section{Approach}

Initial data analysis occurred independently within each Lab; however, similar strategies were employed. Transcribed interviews were imported into qualitative data analysis software ${ }^{29,30}$ to facilitate data management, codebook development, team coding, and intercoder agreement calculations (set at $80 \%$ for both Labs). Within each Lab, an initial set of 
deductive codes was derived from interview questions, evaluation objectives, and review of the literature. Through open coding of transcripts and regular team coding meetings, each Lab produced a codebook consisting of definitions, inclusion and exclusion criteria, and examples. Members of the qualitative team from each Lab used the resulting codebook to complete initial coding.

The authors reviewed coded data from both Labs using a constant comparative approach, ${ }^{31}$ with a focus on identifying resources needed to support PACT implementation and illustrations of how presence or absence of these resources affected ability to delegate within teams. Through a series of regular phone meetings, we identified factors common across each VISN to develop a multi-level framework describing resources required to support successful, sustained implementation and team functioning from the perspectives of PACT pilot teams. Figure 1 provides additional details concerning data collected and analyzed by each Lab separately, as well as our approach to cross-Lab data analysis.

\section{FINDINGS}

We identified four key team-level resources that facilitated ability of primary care staff to work as effective, engaged

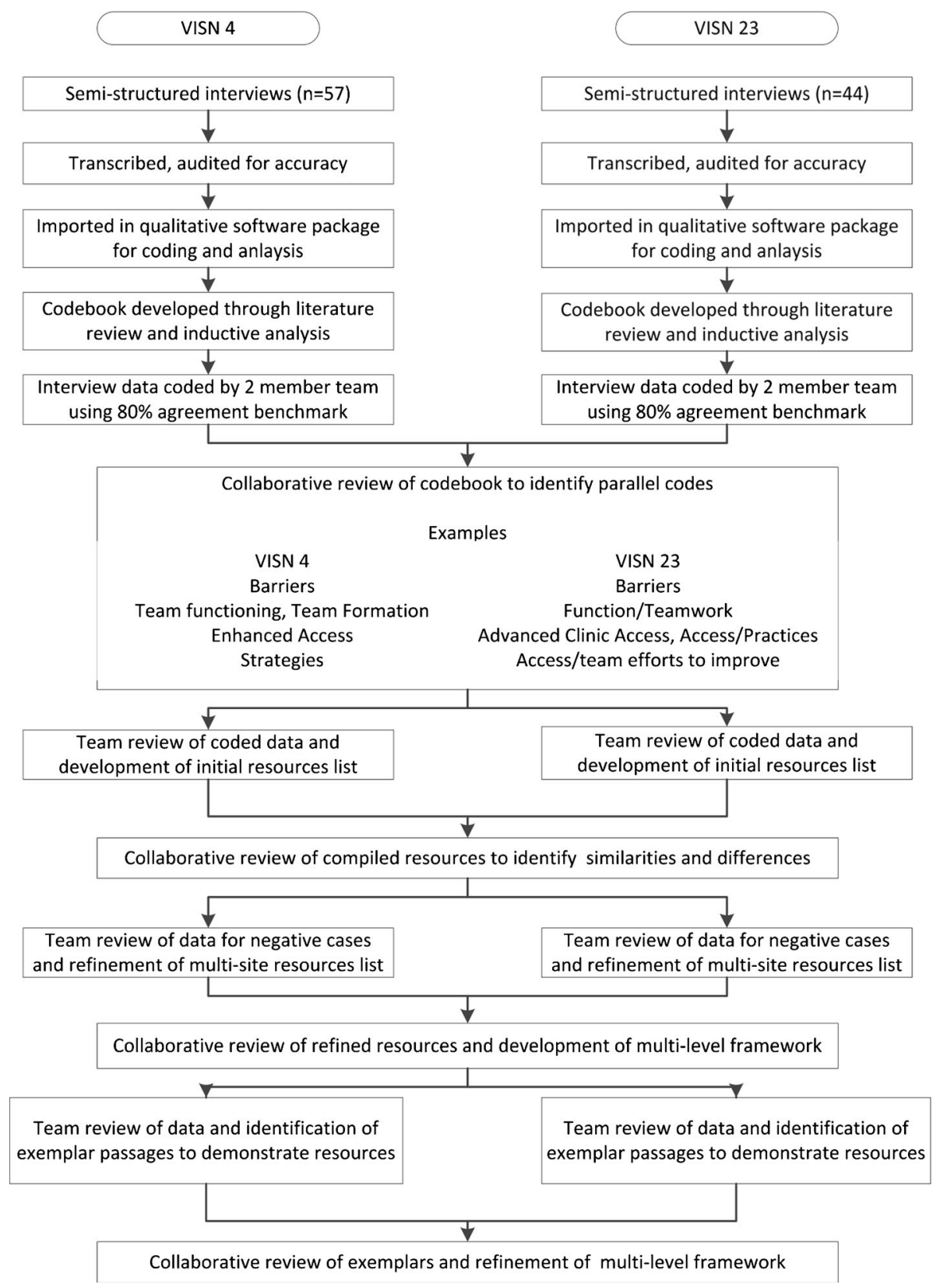

Figure 1. Data analysis procedures. 
teams; conversely, the absence of these resources resulted in barriers to team functioning. Below, we define each resource and characterize how presence or absence of these team-level resources facilitated or impeded effective team functioning for VHA pilot teams. Representative quotes are provided at the end of each subsection in Text Boxes 1-4.

1. Demarcated boundaries and collective identity

An essential contributor to successful team functioning was the ability of staff to identify themselves as belonging to a discrete team with identifiable members. Staff emphasized the importance of stable, consistent team membership, as well as having plans in place to ensure coverage of tasks and responsibilities during short-term or long-term absences of a team member. Willingness to delegate responsibilities for patient care to other team members required a level of certainty about team membership, continuity, and stability.

Clinically licensed members of the primary care team who did not perceive themselves as being part of a team that was well-defined and stable were resistant to delegating patient care tasks to others. Having too few members on a team, lacking team members who fulfilled specific roles, or having team members pulled away to do work outside the team were situations that eroded team effectiveness and created doubt among remaining team members. For example, PCPs recognized that many patient care tasks could potentially be delegated to someone in a different role; however, without a specific RNCM or Clinical Associate assigned to their team on a consistent basis, PCPs were reluctant to delegate any aspects of patient care and perceived themselves as solely responsible for their panel of patients. Lack of clear team membership also created barriers to team members working to the top of their license, as for example when RNCMs on teams lacking a licensed practical nurse or medical support assistant spent the bulk of their time taking vital signs and rooming patients to keep the clinic flow going.

Text Box 1. Team has demarcated boundaries and collective identity, examples
Presence
"I work with my provider and [another] LPN and $R N$. If something has to be done, and I can't do it, I'll say, 'Hey, can you do this?' and vice versa. We have [a] good working team."(Clinical Associate VISN 23)
"I think knowing what's going on gives everybody a sense that we're all the same team. Psychologically, it puts us on a better mindset right from the get-go versus everybody being in their own corner."(Clinical Associate VISN 4)
Absence
"Well, that's pretty hard [to develop a sense of team] when you rotate nurses. You can't really do it. So the provider feels like they're really taking care of their own panel and they don't have much assistance doing it." (PCP VISN 23)
"Telling me I can't have a team... I understand where they're coming from, but I said, 'Well, can't we do it even 15 percent of the time? Can't you say so and so will be my LPN whenever she can?' Without that, how can I do [PACT]?" (PCP VISN 4)

2. Shared goals and sense of purpose

Staff on pilot teams functioned best when they had a cohesive identity from which they derived a sense of common purpose regarding goals for PACT implementation. Individual team members emphasized this common sense of purpose when discussing how they and other members of their team were invested in and believed in the PACT model as a means to improve team functioning and patient care. Shared team goals and values were developed in a number of ways, including exposure to PACT training, writing a team mission statement, or collaborative problem-solving during team meetings. Having shared goals and sense of purpose fostered interdependence on teams; care providers were able to enact patient hand-offs to other members of the team confident that all members of the team were "on the same page".

When components of this shared purpose were missing, respondents experienced an environment of mistrust that served to undermine development of team aims and within-team task delegation. Teams found themselves stalled for development, leading to frustration among both those members who were adamant PACT supporters and those who either had not yet been trained or lacked the resources for complete implementation. Many teams had PCPs or RNCMs who were resistant to delegating work to other team members out of the fear that patient safety or quality of care would be at risk. 
Text Box 2. Team has shared goals and sense of purpose, examples

\begin{abstract}
Presence
"You know we really did all embrace [PACT]. We talked about the different things that would happen for the patients and make it better for them. And even [make it] less stressful for the providers and the nurses, by assuming our roles a little bit differently. We all are on the same page." (RNCM VISN 4)

"It's very rewarding to go to these [trainings] because you get so energized... you want to get back and do these changes. We can work as a team together, see what we can do as a team, and go from there." (Clinical Associate VISN 23)
\end{abstract}

\section{Absence}

"The PACT team stuff... is just not happening. You've got to have everybody buy into it, and if you don't, it isn't going to work. It takes one person to screw it up, and we've got more than one person screwing it up." (PCP VISN 23)

"Part of the problem is that we have a lot of people that have been here forever and ever, and [say] 'This is the way we've always done it!' There's no room for new ideas." (RNCM VISN 23)
3. Mature and open communication characterized by psychological safety

Mature and open communication characterized by psychological safety, a key element supporting team functioning, was exemplified by teams having established, regular practices for exchanging information efficiently and effectively in an environment that promoted trust and encouraged members to voice opinions without fear of repercussions or ridicule. Maintaining multiple lines of communication such as daily huddles, regular team meetings, and means for triaging patient calls to appropriate team members reinforced successful team performance. At the same time, staff expressed the importance of a team atmosphere that allowed for all team members to communicate across professional boundaries and traditional hierarchies.

Respondents who reported working on such teams reflected more positive attitudes toward team membership, where open communication practices facilitated delegation within the team and reinforced trust in team members' abilities through a sense of unity. On the other hand, respondents from teams with poor communication strategies reported challenges with accomplishing tasks due to confusion over responsibilities and feelings of isolation. These respondents were often frustrated with feelings of not being heard or not having opportunities to provide input regarding team-level activities.

Text Box 3. Team engages in mature and open communication, examples

\begin{abstract}
Presence
"You have a weekly meeting... and there's more communication between everybody...you can talk about any sort of issues that are going on. And [PCPs/RNCMs] get a better appreciation of what we do because they're talking about certain issues and it's like, 'Oh yeah, I take care of that,' or 'I could do that.' And they realize more what [we] actually [do]."(Clerical Associate VISN 23)

"We have huddles a lot, and we all have a pretty open relationship with each other. We can say 'This isn't working, can we try this?' So the providers, the nurses, and the clerks, we have all been really working together." (RNCM VISN 4 )
\end{abstract}

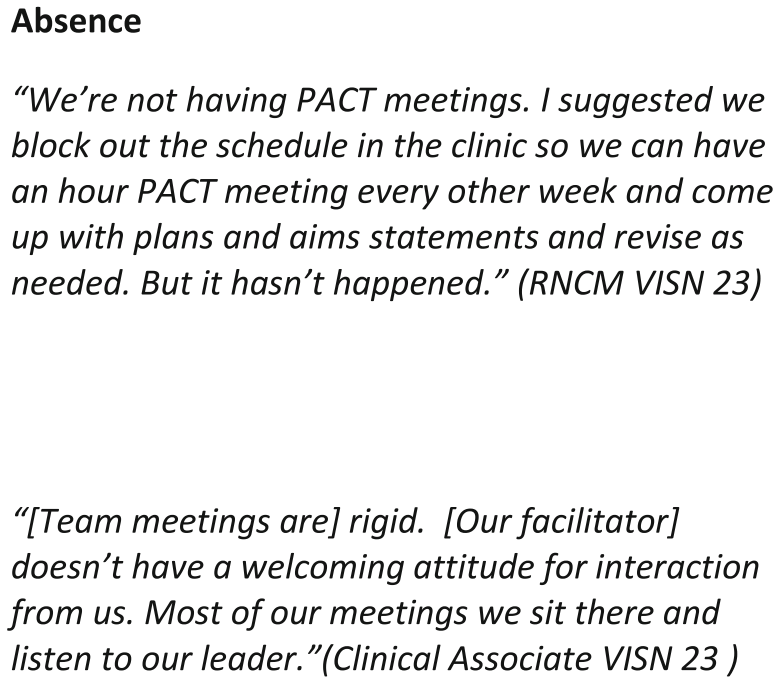
block out the schedule in the clinic so we can have an hour PACT meeting every other week and come up with plans and aims statements and revise as needed. But it hasn't happened." (RNCM VISN 23)

"[Team meetings are] rigid. [Our facilitator] doesn't have a welcoming attitude for interaction from us. Most of our meetings we sit there and listen to our leader."(Clinical Associate VISN 23 ) 
4. Ongoing and intentional role negotiation and development Effective PACTs reallocated tasks to assure that all team members were working at the top of their capabilities. Reallocation of tasks required higher skilled team members such as PCPs and RNCMs to delegate tasks to other team members such as Clinical and Clerical Associates. Although teams were required to follow guidelines associated with licensure and accepted practice, successful team functioning was facilitated by members cooperating internally to determine which tasks could be carried out by whom within the team. Teams who engaged in this process had members who were cognizant of the skills they and others brought to the team, and reflected on this in thinking about what roles they and others could fulfill. Team members were able to openly negotiate task assignments, while recognizing that the process had to be ongoing as individual team members developed new capabilities ranging from improved delegation to basic technical skills.

However, other respondents reported they did not feel they were part of a team, despite having been officially assigned to a PACT. These teams 'in name only' were typified by having waited for external leaders or guidance to define specific team roles and responsibilities. Lack of clear within-team role definition and unclear performance expectations contributed to a team environment where PCPs and RNCMs felt overworked while Clinical and Clerical Associates reported that they felt unappreciated and unable to reach their fullest potential in terms of contributing to the team.

Text Box 4. Team engages in ongoing, intentional role negotiation, examples

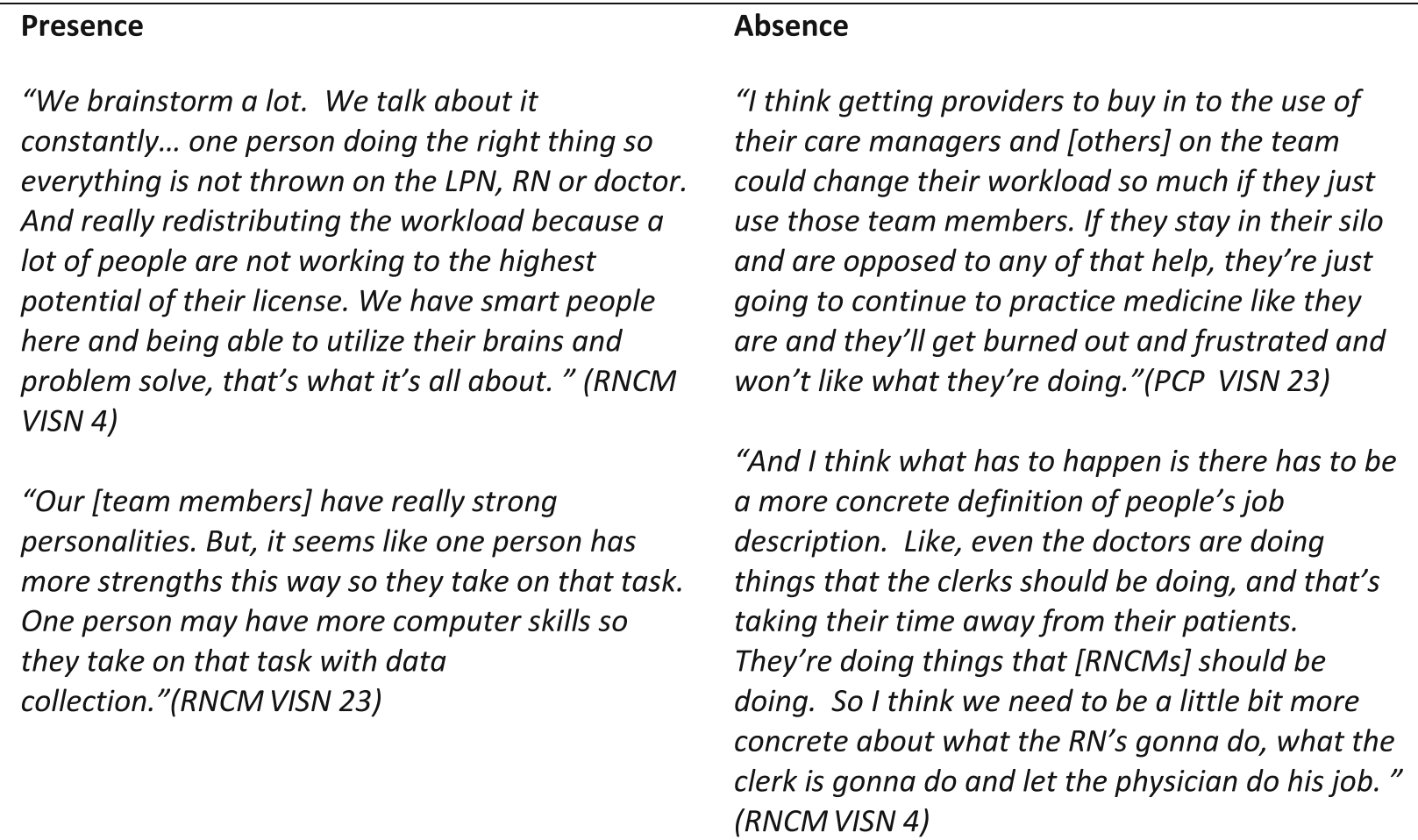

\section{CONCLUSIONS}

The shift toward a team-based model of care involves an evolution of roles and functioning, which is itself transformational. Organizations must go beyond merely communicating the expectation that staff will function as teams by providing essential resources that enable individuals to work in teams. ${ }^{20}$ Successful team functioning is not a point-intime occurrence; it is iteratively produced through new practices over time. Change initiatives that rely on team-based task delegation but fail to provide sufficient resources to support team functioning are unlikely to be successful when it comes to full and sustained implementation. ${ }^{32,33}$

Our cross-lab analysis of interviews with members of VHA pilot teams resulted in development of a framework to describe resources that must be in place to support team formation, successful team functioning, and initiation and sustainment of within-team delegation-all of which are key to PCMH implementation. In the current paper, we report on team-level resources. These findings build on our earlier work describing key resources at the system and clinic levels that facilitated PACT implementation. Namely, 


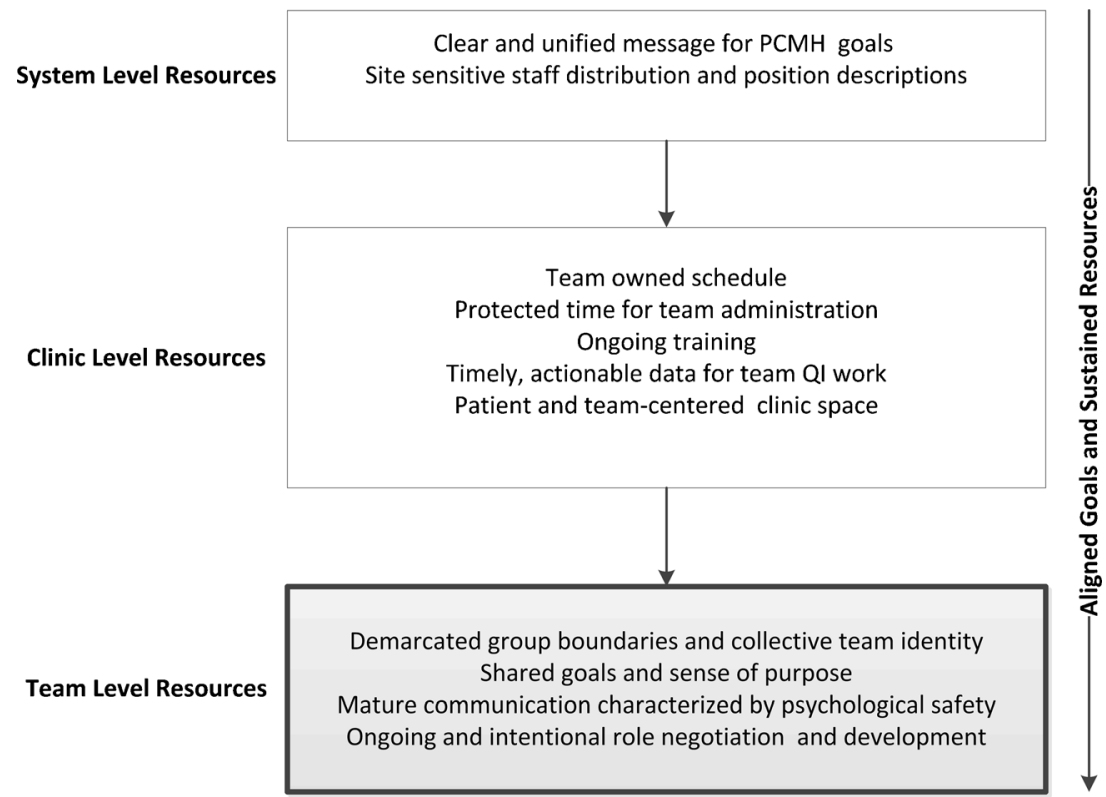

Figure 2. Framework for understanding resources essential for effective medical home teams.

in that earlier work we demonstrate how leadership at the organizational level must provide a clear and consistent message about the goals of quality improvement initiatives such as PCMH, as well as staffing and positions that align with these goals. $^{26,27}$ Without this alignment and sustainment of resources, PACT team members are likely to view QI-related work as low priority in the face of competing demands and time pressures of daily clinic work. ${ }^{19}$ Likewise, teams attempting to change long-standing processes of patient care-for example, matching each patient's appointment needs to the appropriate team member - require support at the clinic-level, often in the form of autonomy over clinic schedules and spaces, administrative time, and ongoing training for team members taking on new responsibilities. Without some 'breathing room' to identify and test out strategies for change, primary care clinics will continue to operate around the pressure on PCPs to see patients face-toface rather than making the transition to a team model of care. ${ }^{34,35}$ At the team-level, our data reveal that in the absence of a well-defined, stable team with shared goals and clear role definitions, PCPs perceived little payoff to changing their patient care routines. Likewise, developing the habit of working as a team was all but impossible for teams lacking regular lines of communication that empowered members to contribute ideas. Figure 2 presents our framework for understanding the relationship among these system-level, clinic-level, and team-level resources, with particular attention to how such resources function to support teamwork and within-team delegation.

Our study has several limitations. The pilot teams we studied were operating within the context of VHA; some constraints experienced by teams, particularly around efforts to redefine roles and position descriptions for non-providers, may not be relevant to primary care staff outside VHA. Our qualitative study was not designed to identify successful teams using performance metrics, nor did we set out to measure variations in teams' ability to carry out team-based task delegation. The analysis reported here relies solely upon semi-structured interview data. While these data provide an expedient mechanism for gathering a broad range of perspectives in a short time, observational methods may speak more directly to the nature, frequency, and paths through which team delegation occurs. Finally, interviews are point-in-time measures that do not reflect the evolution of team functioning over time. Despite such limitations, interview data from each Lab were markedly similar. Repeated assessment for negative cases yielded no outliers, nor a great deal of variance by VISN. Our cross-Lab collaboration demonstrates one approach to strengthening generalizability and transferability of qualitative findings regarding implementation and impact of the PCMH model. ${ }^{36}$ With an increasing number of organizations adopting elements of the PCMH model, and the concomitant evaluation studies, it is essential to provide policy-makers, administrators, and front-line staff with a full picture of the challenges and successes of this model of care, which is relevant across a variety of settings. ${ }^{7,8}$

In summary, we have presented a framework for understanding key resources needed to support effective, engaged teams in PCMH implementation. Our work has a number of potential applications. Our findings can inform an organizational assessment prior to undertaking a transformation to team-based medical care or to identifing organizational assets already in place as well as those in need of improvement. In the case of the VHA PACT initiative, our research expands the understanding of facilitators and impediments to working in teams, and points to potential areas of greatest leverage for supporting team functioning as PACT implementation moves 
beyond pilot teams. For teams that have already started to make PACT-related changes to processes of care, but are struggling, this framework indicates key areas where they may benefit from additional support going forward.

\section{Acknowledgments:}

Contributors: GT and MP would like to acknowledge the contributions of the leadership and staff of the VISN 4 Center for Evaluation of Patient Aligned Care Teams (CEPACT), members of the CEPACT Advisory Board and the VISN 4 PACT Steering Committee, and staff at the VISN 4 Medical Facilities. GLS, ML, and SLS would like to acknowledge the support of the VISN 23 Patient Aligned Care Team Demonstration Laboratory. Additional support comes from the Center for Comprehensive Access \& Delivery Research and Evaluation (CADRE), Department of Veterans Affairs, Iowa City VA Health Care System, Iowa City, IA. We also wish to acknowledge technical assistance from the VA Midwest Health Care Network, Primary Care and Specialty Medicine Service Line and the VISN 23 PACT Collaborative teams. Finally, we are grateful for the comments from the two anonymous reviewers and special issue guest editor.

Funders: The VISN 4 and VISN 23 Demonstration Laboratories are funded by the VA Office of Patient Care Services.

Conflict of Interest: None of the authors have any conflicts of interest related to this study. The views expressed in this article are those of the authors and do not necessarily reflect the position or policy of the Department of Veterans Affairs or the United States government.

Corresponding Author: Gala True, PhD; VISN 4 Center for Evaluation of Patient Aligned Care Teams (CEPACT), Philadelphia Veterans Affairs Medical Center, Building 4100, University and Woodland Aves, Philadelphia, PA 19104, USA (e-mail: Jennifer.True2@va.gov).

\section{REFERENCES}

1. Chin MH, Clarke AR, Nocon RS, et al. A roadmap and best practices for organizations to reduce racial and ethnic disparities in health care. J Gen Intern Med. 2012;27(8):992-1000.

2. Colwill JM, Cultice JM, Kruse RL. Will generalist physician supply meet demands of an increasing and aging population? Health Aff (Millwood). 2008;27(3):w232-41.

3. Bodenheimer T, Chen E, Bennett HD. Confronting the growing burden of chronic disease: can the U.S. healthcare workforce do the job? Health Aff (Millwood). 2009;28(1):64-74.

4. King J, Moulton B. Group Health's participation in a shared decisionmaking demonstration yielded lessons, such as role of culture change. Health Aff (Millwood). 2013;32(2):294-302

5. Jaén CR, Ferrer RL, Miller WL, et al. Patient outcomes at 26 months in the patient-centered medical home national demonstration project. Ann Fam Med. 2010;8(Suppl 1):s57-67.

6. Schram AP. The patient-centered medical home: transforming primary care. Nurse Pract. 2012;37(4):33-9.

7. Peikes D, Dale S, Lundquist E, Genevro J, Meyers D. Building the Evidence Base for the Medical Home: What Sample and Sample Size do Studies Need? Rockville, MD: White Paper, AHRQ; 2011:1-56.

8. Peikes D, Zutshi A, Genevro JL, Parchman ML, Meyers DS. Early evaluations of the medical home: building on a promising start. Am J Manage Care. 2012;18(2):105-16.

9. Bitton A, Schwartz GR, Stewart EE, et al. Off the hamster wheel? Qualitative evaluation of a payment-linked Patient-Centered Medical Home (PCMH) Pilot. Milbank Q. 2012;90(3):484-515.

10. Grumbach K, Grundy P. Outcomes of Implementing Patient Centered Medical Home Interventions: A Review of the Evidence from Prospective
Evaluation Studies in the United States. Patient-Centered Primary Care Collaborative: Washington, D.C; 2010.

11. Raskas RS, Latts LM, Hummel JR, Wenners D, Levine H, Nussbaum SR. Early results show WellPoint's Patient-Centered Medical Home Pilots have met some goals for costs, utilization, and quality. Health Aff (Millwood). 2012;31(9):2002-9.

12. Alexander JA, Bae D. Does the patient-centred medical home work? A critical synthesis of research on patient-centred medical homes and patient-related outcomes. Health Serv Manag Res. 2012;25(2):51-9.

13. Boult C, Reider L, Leff B, et al. The effect of guided care teams on the use of health services results from a cluster-randomized controlled trial. Arch Intern Med. 2011;171(5):460-6.

14. Fishman PA, Johnson EA, Coleman K, et al. Impact on seniors of the patient-centered medical home: evidence from a pilot study. Gerontologist. 2012;52(5):703-11.

15. Reid RJ, Fishman PA, Yu O, et al. Patient-Centered Medical Home Demonstration: a prospective, quasi-experimental, before and after evaluation. Am Manag Care. 2009;15(9):E71-87.

16. Martsolf GR, Alexander JA, Shi YF, et al. The patient-centered medical home and patient experience. Health Serv Res. 2012;47(6):2273-95.

17. Crabtree BF, Chase SM, Wise CG, et al. Evaluation of patient centered medical home practice transformation initiatives. Med Care. 2011;49(1):10-6.

18. Bitton A, Martin C, Landon BE. A nationwide survey of patient centered medical home demonstration projects. J Gen Intern Med. 2010;25(6):584-92.

19. Nembhard IM, Edmonson AC. Making it safe: the effects of leader inclusiveness and professional status on pscyhological safety and improvement efforts in healthcare teams. J Organ Behav. 2006;27(7):941-66.

20. Bodenheimer T, Laing BY. The teamlet model of primary care. Ann Fam Med. 2007;5(5):457-61.

21. Fandre MN. The promises and perils of the MHS' implementation of the patient-centered medical home. Mil Med. 2012;177(12):1445-7.

22. Altschuler J, Margolius D, Bodenheimer T, Grumbach K. Estimating a reasonable patient panel size for primary care physicians with teambased task delegation. Ann Fam Med. 2012;10(5):396-400.

23. Saba GW, Villela TJ, Chen E, Hmmer H, Bodenheimer T. The myth of the lone physician: toward a collaborative alternative. Ann Fam Med. 2012;10:169-73.

24. Mitchell P, Wynia M, Golden R, et al. Core Principles \& Values of Effective Team-Based Health Care. Institute of Medicine: Washington, DC; 2012.

25. Rosland A-M, Nelson $\mathbf{K}$, Sun $\mathbf{H}$, et al. The patient-centered medical home in the Veterans Health Administration. Am J Manage Care. 2013;119(7):263-72.

26. Solimeo SL, Hein M, Paez M, Ono S, Lampman M, Stewart GL. Medical homes require more than an EMR and aligned incentives. Am J Manage Care. 2013;19(2):132-40.

27. True G, Butler AE, Lamparska BG, et al. Open access in the patientcentered medical home: lessons from the Veterans Health Administration. J Gen Intern Med. 2013;28(4):539-45.

28. Solimeo SL, Ono SS, Lampman M, Paez M, Stewart GL. The empowerment paradox as a central challenge to primary care medical home implementation. ND

29. ATLAS.ti [computer program]. Version 6.12. Berlin, Germany: Scientific Software Development; 1999.

30. MAXQDA 10 Software for qualitative data analysis [computer program]. Berlin, Germany: VERBI GmbH; 1998-2012.

31. Parry KW. Constant comparison. In: Lewis-Beck MS, Bryman A, Liao TF, eds. The SAGE Encyclopedia of Social Science Research Methods. Thousand Oaks, CA: SAGE Publications, Inc.; 2004.

32. Kaissi A, Kralewski J, Curoe A, Dowd B, Silversmith J. How does the culture of medical group practices influence the types of programs used to assure quality of care? Health Care Manag Rev. 2004;29(2): $129-38$.

33. Shojania KG, Ranji SR, Shaw LK, et al. Closing the Quality Gap: A Critical Analysis of Quality Improvement Strategies (Vol. 2: Diabetes Care). Rockville, MD: AHRQ; 2004.

34. Nutting PA, Miller WL, Crabtree BF, Jaén CR, Stewart EE, Stange KC. Initial lessons from the first national demonstration project on practice transformation to a patient-centered medical home. Ann Fam Med. 2009; 7(3):254-60.

35. Chesluk BJ, Holmboe ES. How teams work-or don't-in primary care: a field study on internal medicine practices. Health Aff (Millwood). 2010;29(5):874-9.

36. Huberman AM, Miles MB. The Qualitative Researcher's Companion. Thousand Oaks, CA: SAGE Publications, Inc; 2002. 